\title{
Table and Chart
}

\section{Table}

5.1 Ie no hikari of the 1930s 175

\section{Chart}

5.1 Number of advertising pages in Ie no hikari 176 
This page intentionally left blank 